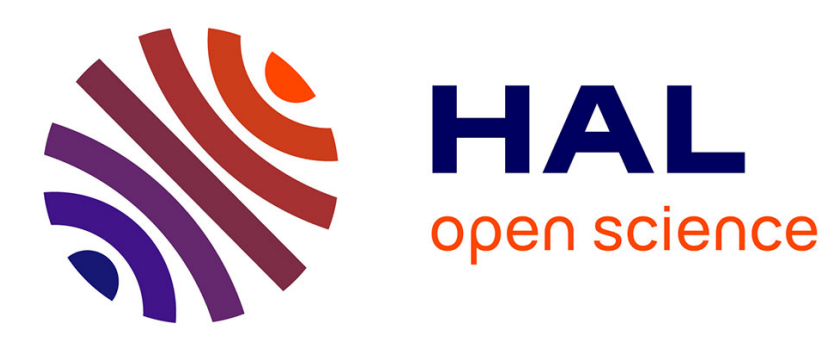

\title{
Sleep Activity Recognition using Binary Motion Sensors
} Yassine El Khadiri, Gabriel Corona, Cédric Rose, François Charpillet

\section{To cite this version:}

Yassine El Khadiri, Gabriel Corona, Cédric Rose, François Charpillet. Sleep Activity Recognition using Binary Motion Sensors. ICTAI 2018 - 30th International Conference on Tools with Artificial Intelligence, IEEE, Nov 2018, Volos, Greece. hal-01943463

\section{HAL Id: hal-01943463 https://hal.inria.fr/hal-01943463}

Submitted on 3 Dec 2018

HAL is a multi-disciplinary open access archive for the deposit and dissemination of scientific research documents, whether they are published or not. The documents may come from teaching and research institutions in France or abroad, or from public or private research centers.
L'archive ouverte pluridisciplinaire HAL, est destinée au dépôt et à la diffusion de documents scientifiques de niveau recherche, publiés ou non, émanant des établissements d'enseignement et de recherche français ou étrangers, des laboratoires publics ou privés. 


\title{
Sleep Activity Recognition using Binary Motion Sensors
}

\author{
Yassine El-Khadiri ${ }^{1,2}$, Gabriel Corona ${ }^{1}$, Cédric Rose ${ }^{1}$, and François Charpillet $^{2}$ \\ ${ }^{1}$ Diatélic, Pharmagest, name.surname@diatelic.fr \\ ${ }^{2}$ Université de Lorraine, CNRS, Inria, LORIA F-54000 Nancy, France, \\ name.surname@inria.fr
}

Monday $5^{\text {th }}$ November, 2018

\begin{abstract}
Early detection of frailty signs is important for the senior population that prefers to keep living in their homes instead of moving to a nursing home. Sleep quality is a good predictor for frailty monitoring. Thus we are interested in tracking sleep parameters like sleep wake patterns to predict and detect potential sleep disturbances of the monitored senior residents. We use an unsupervised inference method based on actigraphy data generated by ambient motion sensors scattered around the senior's apartment. This enables our monitoring solution to be flexible and robust to the different types of housings it can equip while still attaining accuracy of 0.94 for sleep period estimates.
\end{abstract}

Index Terms - Inference mechanisms, Unsupervised learning, Senior citizens, Smart homes, Ambient Assisted Living

\section{Introduction}

The world population is converging towards $17 \%$ being over 60 years old by 2050[1]. Care-taking of this senior population is getting more difficult given the general socio-economic context of the active and senior demographic. From the World bank database, the aged dependency ratio which is the ratio of people older than 64 to the working age population went from $8.6 \%$ in 1960 to $12.9 \%$ in 2016[2]. According to the United States Census Bureau, around $29 \%$ of adults aging 65 or older live alone[3]. The US national median cost of a semi-private Room in a nursing home is projected to grow by $243 \%$ from 2017 to 2047[4]. This paper takes part in a project which goal is to develop an ambient health care monitoring system that minimizes the senior's involvement and interaction with it. The solution should then not need any type of learning or setup to start giving immediate accurate results. We present the algorithm we use for the Sleeping Activity of Daily Living.

Our method works on environments equipped with binary motion sensors. These sensors report motion that happens in their field of view. The sensors we use report a timestamp for when they detect movement and another one for when they no longer detect it. The reported activity data can then be represented by a boolean time-series. Using this representation has many advantages. It is easy to work with but also easy to translate into from more complex representations coming from non binary motion sensors like depth of field cameras for instance.

\section{Related work}

Formal sleep study in the medical field or Sleep medicine is done through Polysomnography[5] which is a diagnostic tool in sleep medicine done in Sleep Labs. However, nowadays, there is a surge of Directto-consumer trackers and devices popularizing and 
bringing perhaps limited but still usefull sleep analysis tools to the masses $[6,7]$. These tools come in different shapes and forms. Some are wearable sensors akin to smart watches $[8,9]$. Others are smartphone applications which come either as companions for the Wearables or as stand-alones[10]. There is also more involved solutions like general public ambient smart-home sensors $[11,12]$ or whole remote Healthcare Monitoring systems[13, 14].

These applications make use of different models and methods to track and follow the user's sleep schedule. Some are based on heartbeat monitoring[15, 16] or accelerometers (actigraphybased solutions)[17], while others use simpler features like smartphone events (screen activations[18, 19], audio features[20], app usage[21]...).

There are three different axes that need exploring to get an overview of these different methods for sleep monitoring: types of sensors, types of collected data, and types of algorithms.

These axes are not totally independent from one another, but they do offer a good framework for setting the context of any given work on this subject.

Following what kind of application is being sought for, sleep tracking can be done using different types of sensors. These can be categorized into two main groups: ambient or wearable. Perhaps the most wellknown ones are of the wearable kind with the big surge of smart-watches and activity tracker wristbands these past few years[6]. However, we are also noticing the same happening for the adoption of ambient smart-home solutions at least on the end consumer market[11]. Ambient sensors are in the middle ground between sleep medicine style studies using Sleep Labs and EEG setups which are aimed at technically advanced and specialized use cases and wearables which are mainly used outdoors during the day.

Ambient sensor setups offer a lot of possibilities in terms of data that can be collected. A simple and straight forward one would be video capture which is often frowned upon and not desirable for obvious privacy concerns. In the context of home automation, motion sensors (PIR sensors) are the most used data sources for activity tracking indoors[22]. These can also come packaged with temperature, light, humidity and other types of sensors in general[23]. Other, perhaps more exotic, sensors allow for capturing 3d body skeletons and silhouettes[24] or heat signatures with radio signals[25].

Algorithms for sleep or ADL tracking in general come in two groups[26]: supervised and unsupervised. Some well-known examples of supervised methods used for human activity recognition are Classifiers like Naive Bayes and Random Forest[27], Hidden Markov Models[28] and Deep Convolutional Neural Networks[29]. Even though the majority of activity recognition or more specifically ambient activity recognition algorithms are supervised, they are inherently hard to scale given not only to their need for annotated training data but also due to inherent differences in the lifestyles of people. Unsupervised methods are mostly model based inference algorithms that can work on a small amount of data. They range from simple rule-based algorithms[18] to Monte-Carlo Markov Chain setups[19].

We propose an ambient assisted living approach that minimizes the patient's involvement with the monitoring system. We make use of motion sensors given their simplicity and availability in conjunction with a model based inference algorithm.

\section{Method}

\subsection{Dataset}

We use one of the many publicly available datasets of the WSU CASAS Project[30] for the experimentation and evaluation of our algorithm. The CASAS project's purpose is to serve as a platform for the collection of real world data for research projects related to smart environments and health monitoring and intervention. The one we used in particular contains data collected from the home of a volunteer adult. The home is equipped with motion sensors that report binary ON and OFF data with a timestamp at each time they sense and stop sensing activity.

which means that we basically have access to a boolean time-series of sensor activations.

The dataset we use has a total of 219 nights worth of data spanning from 2010-11-04 to 2011-06-11 with over $1,713,128$ sensor activations reported from the 
sensors placed all around the resident's house which floor-plan.

Figure 1 shows reported data from these sensors centered around midnight and re-sampled on slices of 10 minutes. As we can see there is an obvious change of rate in sensor activations when the resident is asleep at night. This is the main assumption we make for the elaboration of our algorithm for finding the sleeping periods which we will assume is the interval where we have different sensor activations (in most cases fewer).

The input data for the algorithm for each night inference is centered around midnight and re-sampled on slices of 10 minutes.

\subsection{Algorithm}

\subsubsection{Model}

The main assumption being that the reported sensor data is strongly correlated with the level of activity in the apartment. Periods with very few sensor activations would correlate to calmer activities like Reading or Relaxing, and even longer periods to Sleeping. We also assume that the resident is awake at noon which is most likely the case especially for elder senior residents in nursing homes for example.

Our method is based on the Bayesian Switch-point Model[31], sometimes also known by the name of Bayesian Change Point Analysis: given a sample of observations from a process of randomly occurring events over a period of time, we search for a change in the rate at which events occur. For example an increase or decrease in the frequency of the observations.

We name the period of low activity the Sleep period, and the period of high activity the Wake period.

Since sensor observations $\left(M_{t}\right)$ are boolean time series, they are modeled after a Bernoulli law (1) of parameter $P_{t}$ : the probability that the sensor would activate at the slice $t$. This parameter changes according the period the slice $\mathrm{t}$ is in: Let $P_{s}$ be the probability of observing a sensor activation during the Sleep period and $P_{w}$ of observing a sensor activation during the wake period. Since the goal is to find

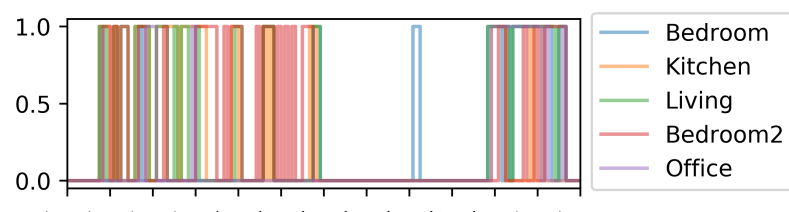

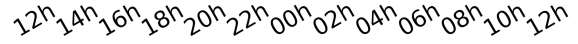

timestamp

Figure 1: Illustration of sensor data centered around midnight showing a clear change in the rate of sensor activations.

when the Sleep period starts and ends we introduce the two variables $T_{s}$ and $T_{w}$ that correspond to its boundaries. This enables us to build a boolean variable $S_{t}$ (2) that conveniently reports which period each time slice $t$ is in.

$$
\begin{gathered}
M_{t} \sim \mathcal{B}\left(P_{t}\right), \quad P_{t}= \begin{cases}P_{s} & \text { if } S_{t}=0 \\
P_{w} & \text { if } S_{t}=1\end{cases} \\
S_{t}=\left\{\begin{array}{lll}
0 & \text { if } \quad T_{s} \leq t<T_{w} \\
1 & \text { if } \quad t<T_{s} \text { or } t \geq T_{w}
\end{array}\right.
\end{gathered}
$$

\subsubsection{Maximum a posteriori estimate}

We consider that we have a stronger prior knowledge of the wake times $T_{w}$ and sleep duration $D=T_{w}-T_{s}$ than on the sleep time $T_{s}$. This way the value of $T_{s}$ is not too much constrained towards its prior.

Inferring $T_{s}$ and $T_{w}$ is done by computing the maximum a posteriori (MAP) estimate $P$ of $T_{s}=t_{s}, T_{w}=$ $t_{w}, P_{s}=p_{s}, P_{w}=p_{w}$ with $\left[t_{s}, t_{w}\right]$ being the sleeping period where a sensor get activated with probability $p_{s}$ and $\left[0, t_{s}[\cup] t_{w}, N\right]$ the wake period where a sensor gets activated with probability $p_{w}$ given the observations of sensor activations $M=\left\{M_{t}, \forall t \in[0, N]\right\}$ :

$$
T_{s}, T_{w}=\underset{T_{s}, T_{w}}{\operatorname{argmax}} \max _{P_{s}, P_{w}} P\left(T_{s}, T_{w}, P_{s}, P_{w} \mid M\right)
$$

with:

$$
\begin{aligned}
P\left(T_{s}, T_{w}, P_{s}, P_{w} \mid M\right) & =P\left(M \mid T_{s}, T_{w}, P_{s}, P_{w}\right) \\
& \times P\left(T_{w}\right) \times P(D)
\end{aligned}
$$


$P\left(M \mid T_{s}, T_{w}, P_{s}, P_{w}\right)$ being the likelihood of $T_{s}=$ $t_{s}, T_{w}=t_{w}, P_{s}=p_{s}, P_{w}=p_{w}$ given the observations $M$.

We consider that $P_{s}$ and $P_{w}$ have a uniform prior meaning that $P\left(P_{s}, P_{w}\right)$ cancels out in the maximization process and we have:

$$
\begin{aligned}
& P\left(T_{s}=t_{s}, T_{w}=t_{w}\right)=\sum_{d} P\left(T_{s}, T_{w}, D=d\right) \\
= & P\left(T_{s}, T_{w}, D=t_{w}-t_{s}\right)+\sum_{d \neq t_{w}-t_{s}} P\left(T_{s}, T_{w}, D\right) \\
= & P\left(T_{w}=t_{w}\right) \times P\left(D=t_{w}-t_{s}\right)
\end{aligned}
$$

As for the $T_{w}$ and $D$ priors we choose a normal distributions centered around 8:00 AM in the morning for $T_{w}$ and 8 hours of sleep for the night duration and both with a standard deviation of an hour and 30 minutes:

$$
\begin{aligned}
T_{w} & \sim \mathcal{N}(8: 00 \mathrm{AM}, 1 h 30) \\
D & \sim \mathcal{N}(8 \text { hours, } 1 \text { h30 })
\end{aligned}
$$

In practice the $T_{w}$ and $D$ values are discretized.

Figure 2 is a graphical representation of the model described above. The slate $t=0, \ldots, N-1$ represents the number of time slices that constitutes the data record spanning from noon of a day $d$ to noon of day $d+1$. The slate $R \in$ Rooms represents the number of sensor timeseries available.

From equation 3 and 4 we have to maximize the posterior:

$$
\underbrace{P\left(T_{w}\right) P(D)}_{\text {prior }} \underbrace{\prod_{t} P\left(M_{t} \mid T_{s}, T_{w}, P_{s}, P_{w}\right)}_{\text {likelihood } \mathcal{L}}
$$

In order to maximize the posterior we have to maximize $\mathcal{L}$ over $P_{s}$ and $P_{w}$ :

$$
\max _{P_{s}, P_{w}} \prod_{\left\{t \mid S_{t}=0\right\}} P\left(M_{t} \mid P_{s}\right) \times \prod_{\left\{t \mid S_{t}=1\right\}} P\left(M_{t} \mid P_{w}\right)
$$

This optimization problem can be split into two:

$$
\max _{P_{s}} \prod_{\left\{t \mid S_{t}=0\right\}} P\left(M_{t} \mid P_{s}\right) \times \max _{P_{w}} \prod_{\left\{t \mid S_{t}=1\right\}} P\left(M_{t} \mid P_{w}\right)
$$

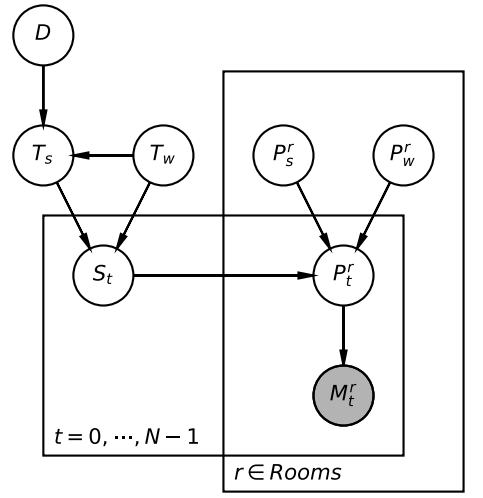

Figure 2: Bayesian network of the sleep inference model

which is:

$$
\max _{p} \prod_{t} P_{p}\left(X_{t}=x_{t}\right) \text { with iid } X_{t} \sim \mathcal{B}(p)
$$

Equation 10 is a Bernoulli maximum likelihood estimate (MLE) for the parameter $p$ that accepts a closed-form solution: $\hat{p}=\frac{\sum x_{t}}{n}$.

Thus our optimization problem becomes:

$$
\underset{T_{s}, T_{w}}{\operatorname{argmax}} \prod_{s=\{0,1\}} \prod_{S_{t}=s} P\left(M_{t} \mid P_{t}=\frac{\sum_{S_{t}=s} M_{t}}{\left|M_{S_{t}=s}\right|}\right)
$$

That can be solved with an exact computation of complexity $\mathcal{O}\left(N^{3}\right)$.

\subsubsection{Soft evidence}

However we have to take into consideration that some sensor observations in some rooms don't give any information related to our model parameters. For example observing a lack of movement doesn't systematically correlate with a passive activity anymore since the resident could be active elsewhere. In other words, our evidence on the observations is no longer certain when taking into consideration all the different sensors scattered in the different rooms. Thus 
we need to introduce soft-evidence in the inference calculations.

Soft-evidence can be modeled over the observations $M$ by the following distribution: $e\left(M_{t} \mid X_{t}\right)[32]$. It isn't a model parameter but an external process we hook into the MAP computation to model the uncertainty of the observations. So we get to freely set the values of $e$ :

\begin{tabular}{c|c|c}
$e$ & $M_{t}=0$ & $M_{t}=1$ \\
\hline$X_{t}=0$ & 0.3 & 0.7 \\
\hline$X_{t}=1$ & 0 & 1
\end{tabular}

The new objective function will be the expectation of the log-posterior:

$$
\int_{M \sim e(X)} \log \left(P\left(T_{w}\right) \times P(D) \times \mathcal{L}\right) \mathrm{d} e(X)
$$

i.e.

$$
\log P\left(T_{w}\right)+\log P(D)+\int \log \mathcal{L}
$$

For a given $T_{s}$ and $T_{w}$, the optimum value of $P_{s}$ is the one that maximizes:

$$
\begin{aligned}
& \int \log P\left(M_{t \mid S_{t}=0} \mid T_{s}, T_{w}, P_{s}, P_{w}\right) \mathrm{d} e(X) \\
& \quad=\log P \sum_{t} E\left[M_{t}\right]+\log (1-P) \sum_{t}\left(1-E\left[M_{t}\right]\right)
\end{aligned}
$$

Which is:

$$
\hat{P}_{s}=\frac{\sum_{t \mid S_{t}=0} E\left[M_{t}\right]}{\operatorname{Card}\left(\left\{t \mid S_{t}=0\right\}\right)}
$$

The same goes for $P_{w}$.

\subsection{Method evaluation}

We evaluate our algorithm using the sleep annotations present in the dataset. We run the inference for each night then compute the error over the inferred values of $T_{s}$ and $T_{w}$ :

$$
e_{x}=T_{x}-T_{x}^{a n n o t a t e d}
$$

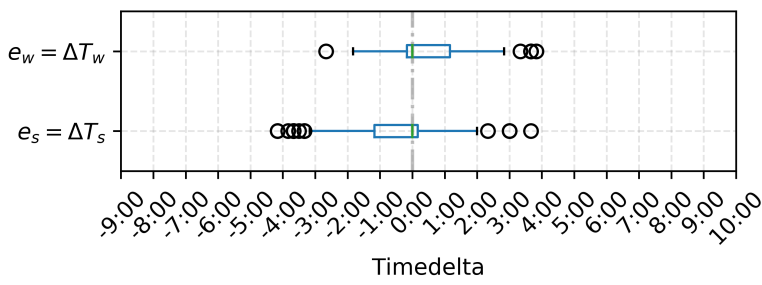

Figure 3: Boxplot of the error on $T_{s}, T_{w}$, and $D$

which is the distribution of the relative error over all of the 219 available nights. A box plot of this distribution gives an idea of how much outliers would be resulted from the algorithm. They are points outside the range $\left[Q_{1}-1.5 \times I Q R, Q_{3}+1.5 \times I Q R\right]$.

We also evaluate it as a binary classification problem for each time slice by constructing the vectors of annotated times $S=\left(S_{t} \mid t \in 0 . . N\right)$ and inferred times $\hat{S}=\left(\hat{S}_{t} \mid t \in 0 . . N\right)$. We can thus compute the accuracy, precision, recall and F1 scores over all the nights.

\section{Results}

The results on the annotated dataset yields a global accuracy 0.94 with an F1 score of 0.90 . The precision and recall scores are respectively 0.85 and 0.95 . Figure 4 shows a more detailed view for these results on an histogram over all of the dataset's nights. From the accuracy histogram, $80.82 \%$ of the evaluated nights have an accuracy over 0.9 . $44.74 \%$ for the precision, $89.5 \%$ for the recall and $56.16 \%$ for the F1 score.

Figure 3 contains the boxplots of the distributions of the relative error on $T_{s}, T_{w}$, and the night duration $D$ rounded on hours. Both medians are centered around 0 minutes. $e_{s}$ whiskers span from -3 hours and 10 minutes to +2 hours while $e_{w}$ 's span from -1 hour 50 minutes to +2 hours 50 minutes. 


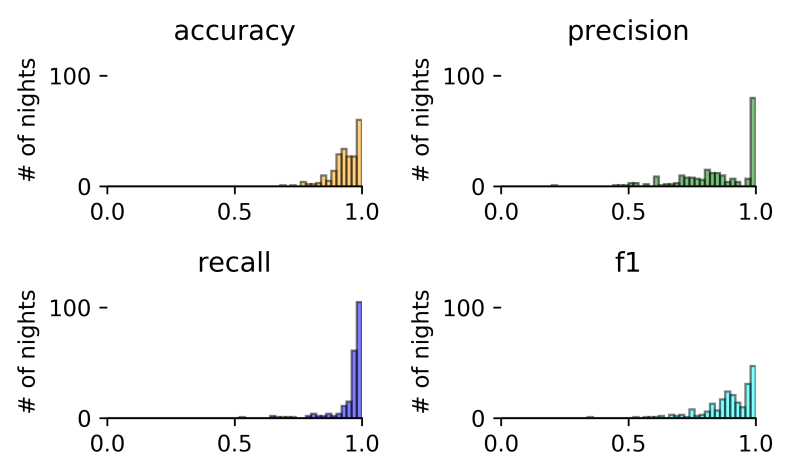

Figure 4: Histogram of the computed accuracy, precision, recall and F1 scores for each night

\section{Discussion}

The main contribution of this work is a method for sleep inference which is computationally inexpensive and applicable to all kinds of ambient sensor setups as long as the reported data holds motor activity information. This method is already directly usable on any new or existing installation as is or with minimum tuning on the $T_{s}$ and $D$ priors. The use of soft evidence makes it possible to work on data without having to take into consideration the resident's whereabouts and possibly have in the mix sensor data not following the sleep-wake model without it impacting too much the inference results.

The accuracy, precision, recall, and F1 global and histogram scores show that this method is also in line with and even slightly better than the expected results from an unsupervised one[19].

SensibleSleep[19] is also a discrete event based method for extracting sleep patterns. The paper uses this method in conjunction with smartphone events. The inference is based on the Markov Chain Monte Carlo method[33] that fits the model to the observed data and outputs the posterior distribution of the parameters. In contrast, we show that by using our model, directly computing a closed form solution yields comparable results while being computationally inexpensive even on smaller bin sizes (our method discretizes the day into 10 minute bins in- stead of 15 minutes for theirs).

Another advantage of this method is the fact that we do not assume $P_{s} \leq P_{w}$ in the inference algorithm since it is not necessarily true all the time. For example chances are that we observe more movements in the bedroom during the night than the day either because of sleep agitation or bathroom visits or sensitive sensors. However this explains outlier cases where the algorithm finds a segment of higher likelihood with more sensor activations than the true sleep period.

There are also other limits to how reliable this algorithm can be, since as shown on figure 3 , the inference seems inclined into overestimating wake times and underestimating sleep times.

A possible enhancement to the algorithm can be to add a learning component on the priors so the more data gets collected the more they get refined. Another one might be a way to compute the confidence in the inferred sleep periods as a starter for work on alerts of sleep agitation or deprivation or unusual and abnormal activity.

However this algorithm in its current state is already deployed in 16 installations including nursing homes and private apartments. The wake time inference is used by the nursing staff as a way to approximate the time when residents wake up so they can go help them in their morning routine. From the inferred sleep period we can also infer nocturnal activity for potentially fragmented sleep problems or medical problems related to frequent bathroom visits.

\section{Conclusion}

We have presented an unsupervised method for sleep period detection using binary time-series of motor activity data. This method is quite simple and computationally inexpensive which makes it possible to integrate into existing medical monitoring devices. It is also cost-effective as it doesn't need any special setup (calibration, learning phase or special sensor placement) that for example depends on the geometry or the nature of the residency's different rooms. The method also supports a dynamically changing number of sensors. This algorithm is currently being 
used in nursing home rooms and apartments for the experimentation phase of the project this paper has originated from.

\section{References}

[1] W. He, D. Goodkind, and P. Kowal, "International population reports - an aging world: 2015," Tech. Rep. P95-16-1, United States Census Bureau, 2016. Available from: https://www. census.gov/content/dam/ Census/library/publications/2016/demo/ p95-16-1.pdf (Visited on 2018-05-03).

[2] W. Bank, "Age dependency ratio, old (from: https: //data.worldbank.org/indicator/ SP.POP.DPND.OL (Visited on 2018-05-14), 2017.

[3] "The majority of children live with two parents, census bureau reports," Tech. Rep. CB16-192, United States Census Bureau, 2016. Available from: https://www. census.gov/newsroom/ press-releases/2016/cb16-192.html (Visited on 2018-05-03).

[4] Genworth, "Cost of care survey 2017 - summary," no. 131168, 2017. Available from: https://www.genworth.com/dam/Americas/ US/PDFs/Consumer/corporate/cost-ofcare/131168_081417.pdf (Visited on 2018-05$03)$.

[5] J. Haba-Rubio and J. Krieger, "Evaluation instruments for sleep disorders: A brief history of polysomnography and sleep medicine," Introduction to modern sleep technology, pp. 19-31, 2012.

[6] "A third of people track their health or fitness," tech. rep., GfK, 2016. Available from: https://www.gfk.com/insights/pressrelease/a-third-of-people-track-theirhealth-or-fitness-who-are-they-and-whyare-they-doing-it/ (Visited on 2018-05-03).

[7] D. Pogue, "What fitbit's 6 billion nights of sleep data reveals about us." Available from: https://finance.yahoo.com/news/ exclusive-fitbits-6-billion-nightssleep-data-reveals-us-110058417.html (Visited on 2018-05-03), 2018.

[8] "Fitbit." https://fitbit.com/sleep-better (Visited on 2018-05-03).

[9] "Biostrap." https://biostrap.com/help/ sleep (Visited on 2018-05-03).

[10] "Sleep cycle alarm clock." https : //sleepcycle.com/how-it-works (Visited on 2018-05-03).

[11] "Sleeptracker." https://sleeptracker.com (Visited on 2018-05-03).

[12] "Beddit." https://beddit.com (Visited on 2018-05-03).

[13] "Keystone care." https:// keystonetechnologies.com/healthsolutions (Visited on 2018-05-03).

[14] "Cormarch remote care for seniors." https:// comarch.com/healthcare/products/remotemedical-care/remote-care-for-seniors (Visited on 2018-05-03).

[15] S. Benedetto, C. Caldato, E. Bazzan, D. C. Greenwood, V. Pensabene, and P. Actis, "Assessment of the fitbit charge 2 for monitoring heart rate," PLOS ONE, vol. 13, pp. 1-10, 02 2018. https://doi.org/10.1371/journal. pone. 0192691 .

[16] T. Coppetti, A. Brauchlin, S. Müggler, A. Attinger-Toller, C. Templin, F. Schönrath, J. Hellermann, T. F. Lüscher, P. Biaggi, and C. A. Wyss, "Accuracy of smartphone apps for heart rate measurement," European Journal of Preventive Cardiology, vol. 24, no. 12, pp. 12871293, 2017. PMID: 28464700, https://doi. org/10.1177/2047487317702044.

[17] L. Hyun-Ah, L. Heon-Jeong, M. JoungHo, L. Taek, I. Hoh, C. Chul-Hyun, and K. Leen, "Comparison of wearable activity 
tracker with actigraphy for sleep evaluation and circadian rest-activity rhythm measurement in healthy young adults," Psychiatry Investigation, vol. 14, no. 2, pp. 179-185, 2017. PMID: PMC5355016, https://www.ncbi.nlm. nih.gov/pmc/articles/PMC5355016/.

[18] S. Abdullah, M. Matthews, E. L. Murnane, G. Gay, and T. Choudhury, "Towards circadian computing: "early to bed and early to rise" makes some of us unhealthy and sleep deprived," Proceedings of the 2014 ACM International Joint Conference on Pervasive and Ubiquitous Computing, pp. 673-684, 2014.

[19] A. Cuttone, P. Bækgaard, V. Sekara, H. Jonsson, J. E. Larsen, and S. Lehmann, "Sensiblesleep: A bayesian model for learning sleep patterns from smartphone events," PLOS ONE, vol. 12, pp. 1-20, 012017.

[20] Y. Ren, C. Wang, J. Yang, and Y. Chen, "Fine-grained sleep monitoring: Hearing your breathing with smartphones," in 2015 IEEE Conference on Computer Communications (INFOCOM), pp. 1194-1202, April 2015.

[21] Z. Chen, M. Lin, F. Chen, N. D. Lane, G. Cardone, R. Wang, T. Li, Y. Chen, T. Choudhury, and A. T. Campbell, "Unobtrusive sleep monitoring using smartphones," in 2013 7th International Conference on Pervasive Computing Technologies for Healthcare and Workshops, pp. 145-152, May 2013.

[22] X. Luo, Q. Guan, H. Tan, L. Gao, Z. Wang, and X. Luo, "Simultaneous indoor tracking and activity recognition using pyroelectric infrared sensors," Sensors, vol. 17, no. 8, 2017.

[23] "Fibaro motion sensors." https://www . fibaro.com/en/products/motion-sensor/ (Visited on 07/05/2018).

[24] A. Dubois and F. Charpillet, "A gait analysis method based on a depth camera for fall prevention," in 2014 36th Annual International Conference of the IEEE Engineering in Medicine and Biology Society, pp. 4515-4518, Aug 2014.
[25] C.-Y. Hsu, A. Ahuja, S. Yue, R. Hristov, Z. Kabelac, and D. Katabi, "Zero-effort in-home sleep and insomnia monitoring using radio signals," Proc. ACM Interact. Mob. Wearable Ubiquitous Technol., vol. 1, pp. 59:1-59:18, Sept. 2017.

[26] P. Rashidi and A. Mihailidis, "A survey on ambient-assisted living tools for older adults," vol. 17, pp. 579-90, 052013.

[27] T. Nef, P. Urwyler, M. Büchler, I. Tarnanas, R. Stucki, D. Cazzoli, R. Müri, and U. Mosimann, "Evaluation of three state-of-the-art classifiers for recognition of activities of daily living from smart home ambient data," Sensors, vol. 15, no. 5, pp. 11725-11740, 2015.

[28] L. Lu, C. Qing-ling, and Z. Yi-Ju, "Activity recognition in smart homes," vol. 76, 112016.

[29] J. B. Yang, M. N. Nguyen, P. P. San, X. L. $\mathrm{Li}$, and S. Krishnaswamy, "Deep convolutional neural networks on multichannel time series for human activity recognition," in Proceedings of the 24th International Conference on Artificial Intelligence, IJCAI'15, pp. 3995-4001, AAAI Press, 2015.

[30] D. J. Cook, "Learning setting-generalized activity models for smart spaces," IEEE intelligent systems, vol. 27, no. 1, pp. 32-38, 2012.

[31] B. A. Maguire, E. Pearson, and A. Wynn, "The time intervals between industrial accidents," Biometrika, vol. 39, no. 1/2, pp. 168-180, 1952.

[32] J. Pearl, Probabilistic Reasoning in Intelligent Systems: Networks of Plausible Inference. San Francisco, CA, USA: Morgan Kaufmann Publishers Inc., 1988.

[33] C. Andrieu, N. De Freitas, A. Doucet, and M. I. Jordan, "An introduction to mcmc for machine learning," Machine learning, vol. 50, no. 1-2, pp. 5-43, 2003. 